\title{
HIGH-RESOLUTION OPTICAL COHERENCE TOMOGRAPHY AND ITS DISPERSION COMPENSATION
}

\author{
Chih-Wei Lu, Meng-Tsan Tsai, Yih-Ming Wang, Chia-Wei Sun, I-Jen Hsu, Su-Feng Chen, \\ Chen-Kuan Lee, Yean-Woei Kiang and C. C. Yang \\ Graduate Institute of Electro-Optical Engineering and Department of Electrical Engineering, \\ National Taiwan University, 1, Roosevelt Road, Section 4, Taipei, Taiwan \\ (phone) 886-2-23657624 (fax) 886-2-23652637 (E-mail) ccy@cc.ee.ntu.edu.tw \\ Chii-Wann Lin \\ Graduate Institute of Bioengineering, National Taiwan University, Taipei, Taiwan
}

\begin{abstract}
We demonstrate a novel method for achieving the axial resolution of an OCT system beyond the limit set by the source spectral width. Then, a few methods for dispersion compensation and image improvement are discussed.
\end{abstract}

Optical coherence tomography (OCT) has caught much attention because of its high-resolution and high-sensitivity scanning capability for biomedical diagnosis. Recently, with the availability of light sources of extremely large bandwidths, axial resolution of around $1 \mu \mathrm{m}$ has been achieved. However, for implementing such high resolution, costly broadband light sources, such as ultra-short solid-state laser and super-continuum generation pumped with a short pulse, are needed. In this paper, we demonstrate a novel approach for reaching high resolution with a light source of a relatively small bandwidth. We can achieve OCT axial resolution beyond the transform limit of light source spectral width. The basic idea of implementing such high OCT resolution is as follows: We varied the offset of the central wavelength ray from the rotation axis, $x_{0}$, in the optical phase delay lines used in our OCT systems. With different $x_{0}$ values, the repeated OCT scans result in different chirps in the interference fringe patterns. The superposition of the repeated scan images leads to an OCT result of high resolution beyond the transform limit of light source spectrum. The OCT system used an SLD with $950 \mathrm{~nm}$ in center wavelength and $40 \mathrm{~nm}$ in spectral width. The theoretical resolution value is around $10 \mu \mathrm{m}$. We used this system to scan an onion sample. Figs. 1 (a)-(c) show the images of a longitudinal scan corresponding to three $x_{0}$ values. The superposition of the three images results in Fig. 2. Here, one can see that the resolution of the onion sample is much improved. In estimation, the axial resolution has been enhanced up to $5 \mu \mathrm{m}$. This is better than the transform limit. OCT resolution beyond the transform limit is useful for building high resolution OCT systems with low cost light sources. However, it requires repeated scans of a sample. Repeated scanning with different $x_{0}$ values may have two implications. First, the scanning time becomes longer. Second, the repeated longitudinal scans require a stable lateral scan system. In the first issue, the longer scanning time may not represent a serious problem when it is compared with that of a super-high-resolution OCT system. Because of the extremely large bandwidth, the widely used optical phase delay line cannot be applied in such an OCT system. The use of other longitudinal scanning mechanisms, such as a constant-speed translation stage, makes the scanning speed about 1000 times slower than that using an optical phase delay line. Therefore, several repeated scans with an optical phase delay line still represent an advantage if the achieved resolution is comparable to that with a light source of extremely broad spectrum.

Acknowledgement

This research was supported by National Health Research Institute, The Republic of China; under the grant of NHRI-EX92-9220EI.

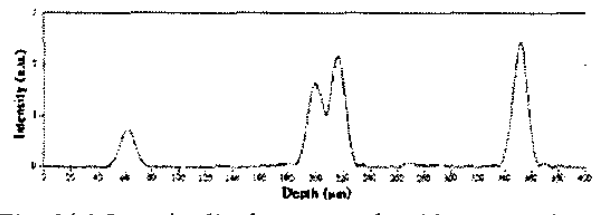

Fig. 1(a) Longitudinal scan result with an $x_{0}$ value.

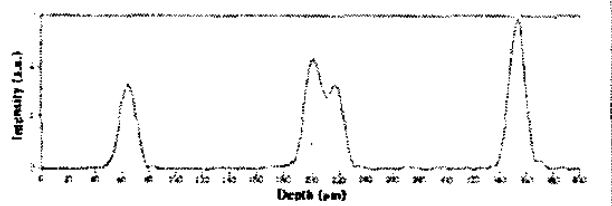

Fig. 1(c) Longitudinal scan result with the third $x_{0}$ value.

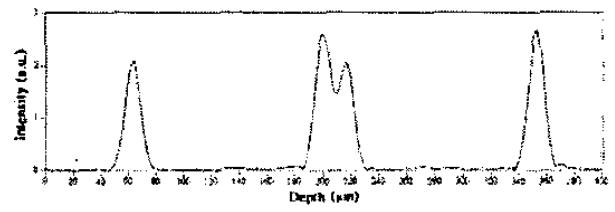

Fig. 1(b) Longitudinal scan result with another $x_{\theta}$ value.

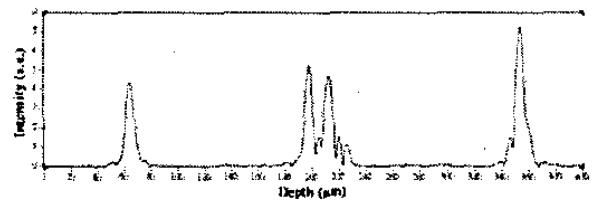

Fig. 2 Superimposed image. 